\title{
A "Performative Turn" in Translation Studies? Reflections from a sociological perspective
}

\author{
Michaela Wolf \\ University of Graz, Austria
}

The increasing interest in the analysis of the link between and interaction of performance and translation has been brought about not least in the wake of the emergence of a sociology of translation. This is particularly due to the emphasis in research on the figure of the translator and other agents involved in the translation process and more precisely to the exploration of the role and function of the translator as a co-subject of the translation performance. The following reflections will focus on the potential epistemological force of the concept of performance with reference both to the social occurrence of translation and to the term's contribution to conceptualizing a wider, perhaps more metaphorically nourished perception of translation. 